\title{
Chronic chikungunya virus musculoskeletal disease: what are the underlying mechanisms?
}

\author{
"With the reemergence of chikungunya in the last decade, there are \\ large numbers of individuals living with chronic \\ musculoskeletal disease. "
}

Mary K McCarthy' \& Thomas E Morrison ${ }^{*, 1}$

First draft submitted: 1 December 2015; Accepted for publication: 4 December 2015; Published online: 4 March 2016

Chikungunya virus (CHIKV) is a positive-sense enveloped RNA virus that causes large epidemics of musculoskeletal disease, including acute and chronic forms of arthritis. Starting in 2005, CHIKV re-emerged to cause outbreaks of unprecedented scale in islands of the Indian Ocean, India, South East Asia, and Europe [1-5]. In 2013, CHIKV reached the Western Hemisphere, where it is responsible for outbreaks in the Americas that have affected millions [6]. Acute CHIKV infection generally presents with fever, severe joint pain, muscle aches and rash, which subside in 7-10 days. However, up to two-thirds of infected individuals experience an incapacitating arthralgia, which often includes signs of joint inflammation and tenosynovitis that persists for months or years after the acute phase $[7,8]$. These persistent disease signs and symptoms cause a substantial economic burden and loss of quality of life [7]. Vaccines and antiviral agents are not currently available for CHIKV infection, and treatment is limited to managing symptoms with analgesics and anti-inflammatory drugs.

Given the high percentage of patients with persistent symptoms following previous CHIKV outbreaks on other continents, it is likely that there will be a substantial population in the Americas with long-lasting pain and disability as a result of chronic CHIKV disease. Initial reports from ongoing outbreaks indicate that chronic disease is already prevalent in parts of the Americas [9-11]. Despite this growing public health concern, the underlying mechanisms of chronic musculoskeletal pain and inflammation following acute CHIKV infection are not well understood. The factors that contribute to the development of chronic CHIKV disease in individual patients are complex and may include: genetic predispositions; pre-existing arthropathy or other co-morbidities; direct virus-induced tissue damage; longterm persistence of CHIKV infection in tissues with concomitant inflammation; and activation of autoimmune responses. Thus, more research in humans and animal models is needed, as identifying effective therapies for the wide spectrum of chronic CHIKV disease manifestations will be challenging without an improved understanding of the molecular mechanisms that contribute to chronic CHIKV disease pathogenesis.

'Department of Immunology \& Microbiology, University of Colorado School of Medicine, CO 80045, USA

*Author for correspondence: Tel.: +1 303 724 4283; Fax: +1 303 724 5226; thomas.morrison@ucdenver.edu

“...in patients without pre-existing conditions, the cause of chronic chikungunya disease is less clear." 
"There is a critical need to improve models that measure and evaluate chronic chikungunya disease manifestations."
Long-term studies of patients following the 2005-2006 CHIKV outbreak on La Reunion Island identified several risk factors for chronic CHIKV disease, including increased symptom severity during the acute phase, age ( $>45$ years older), sex (female), or a pre-existing rheumatological condition [12]. Thus, in some patients there is evidence that CHIKV infection exacerbated pre-existing chronic arthropathy. However, in patients without pre-existing conditions, the cause of chronic CHIKV disease is less clear. Two main categories of chronic CHIKV disease have been described: post-CHIKV musculoskeletal disorders and post-CHIKV de novo chronic inflammatory rheumatisms (CIR) [13]. The former have been managed with analgesics, anti-inflammatory drugs, and physiotherapy. Post-CHIKV de novo CIR include rheumatoid arthritis (RA), spondyloarthritis, and undifferentiated polyarthritis and occur in patients without a history of an articular disorder prior to CHIKV infection.

\section{Evidence for autoimmune responses \& viral persistence during chronic CHIKV disease}

There is little evidence of systemic autoimmunity during acute or chronic CHIKV disease. Autoreactive $\mathrm{CD}^{+}{ }^{+}$or $\mathrm{CD} 8^{+} \mathrm{T}$ cells have not been described in CHIKV patients. Serum levels of rheumatoid factor (RF), antinuclear antibodies (ANA), and anticitrullinated peptide antibodies (anti-CCP) are not appreciably elevated in patients with joint disease after acute CHIKV infection [14-17]. In studies directly comparing patients with chronic disease to those having recovered, no association between disease and ANA levels was detected [14,16]. However, it is possible that autoantigens or autoreactive lymphocytes could be present in local joint or muscle tissue and contribute to chronic CHIKV disease in these patients. In a subset of patients that have been diagnosed with post-CHIKV CIR, 30-67\% are positive for ANA, RF or anti-CCP, suggesting some autoimmune involvement $[13,18,19]$. It is unclear whether these patients have a genetic predisposition to RA triggered by CHIKV infection, or whether post-CHIKV RA is a unique disease. Moreover, it is also unclear whether autoantibody responses in this patient subset contribute to chronic disease or simply associate with it.

Assays for infectious virus, viral RNA, or viral antigen in tissues of patients with chronic CHIKV disease are not routinely performed. In a limited number of studies, CHIKV antigens and RNA have been detected in synovial or muscle tissue biopsies collected from patients with chronic musculoskeletal pain; CHIKV antigen is detected in perivascular macrophages in some cases. More definitively, experiments using nonhuman primates and mice have demonstrated that CHIKV RNA and antigen remain detectable in musculoskeletal and other tissues for months after infection [20-22]. In mice, the long-term persistence of viral RNA is associated with chronic inflammatory pathology and immune activation. Peripheral blood mononuclear cells (PBMCs) isolated from patients with chronic CHIKV disease express elevated levels of IFN- $\alpha$ [23]. Additionally, persistent CHIKV capsid protein and mRNA present in the synovium of a patient with severe chronic arthralgia was associated with IFN- $\alpha$ expression, high levels of apoptosis, and synovial tissue injury. This supports the notion that persistent virus infection or viral products, rather than an autoimmune response, promotes prolonged immune activation and injury of musculoskeletal tissue. If the long-term symptoms of CHIKV infection result from autoimmunity or a hyperactive immune response to persistent viral antigen, immunosuppression might alleviate symptoms. In contrast, if chronic CHIKV disease is a result of persistent viral replication and direct virus-induced cytotoxicity, treatment with immunosuppression might exacerbate symptoms and joint damage. There are reports that immunosuppression by methotrexate or anti-TNF- $\alpha$ blockade produces a positive clinical response in some patients with chronic CHIKV disease, although a subset of patients who received immunosuppressive therapy developed progressive joint or bone damage $[13,18]$. These data highlight the pressing need to investigate further the role of virus persistence in chronic CHIKV disease. This information is essential to inform the use of immunosuppressive agents and guide the development of new therapeutic approaches.

\section{Using animal models to fill the knowledge gaps}

There is a critical need to improve models that measure and evaluate chronic CHIKV disease manifestations. Host genetic variation likely influences the outcome of CHIKV infection and the development of chronic disease. Therefore, it will be important to define mechanisms of chronic CHIKV disease pathogenesis in genetically diverse populations. These studies could take advantage of naturally occurring genetic variations in different inbred and outbred mouse strains or newly created 
populations of genetically diverse mice [24]. In addition, whether CHIKV infection induces autoimmunity and whether autoimmune responses contribute to chronic disease remain unanswered questions. To date, studies using animal models do not support a role for an autoimmune etiology in chronic CHIKV disease; however, as alluded above, these studies have been performed using a narrow set of genetic backgrounds. Animal models representing diverse genetic backgrounds could be employed to test whether autoantibodies or autoreactive lymphocytes are induced systemically or locally at sites of inflammation during the acute and chronic phases of CHIKV infection. If autoimmune responses were detected during CHIKV infection, demonstration that these antiself responses are arthritogenic, that disease is transferable by immune cells or serum in the absence of virus, and that autoimmune responses persist in the absence of infection would be major developments in elucidating the pathogenesis of chronic $\mathrm{CHIKV}$ disease. Additionally, CHIKV infection could be studied using animal models of induced, genetically manipulated, or spontaneous arthritis. If autoimmunity is present and chronic CHIKV disease is exacerbated, such results would provide evidence that: predisposition to a rheumatological disorder contributes to the development and/or severity of chronic CHIKV disease; and autoimmunity may contribute to chronic disease, at least in a subset of patients.

Persistent virus infection could contribute to chronic inflammation and tissue injury. It is clear that persistent CHIKV RNA and antigen are present in musculoskeletal tissue of humans, nonhuman primates, and mice, but whether this is from ongoing low-level replication or cells harboring undegraded viral material requires further investigation. In addition, the cell types infected by CHIKV during acute and chronic infection have not been fully defined. Animal models could be used to determine the distribution of persistent CHIKV RNA and antigen in tissue, and whether CHIKV components are associated with foci of inflammatory cells. Additional experiments could be performed to determine whether the level of persistent CHIKV antigen in musculoskeletal tissue changes over time or in response to certain stimuli such as stress or additional infections. These experiments could provide evidence that unrelated or related disease exacerbations in patients with chronic disease are associated with CHIKV recrudescence in musculoskeletal tissue.

\section{Conclusion}

With the re-emergence of CHIKV in the last decade, there are large numbers of individuals living with chronic musculoskeletal disease. More effort must be devoted to understanding the underlying causes of ongoing symptoms in these patients. It is not clear whether modulation of inflammation by immunosuppressive drugs will be an effective 'catch-all' strategy to limit musculoskeletal signs and symptoms in patients with chronic CHIKV disease or whether specific antiviral agents would mitigate chronic CHIKV disease. The varied spectrum of clinical presentation and response to therapy suggests that the underlying causes of ongoing symptoms may differ from patient to patient. An improved understanding of the molecular mechanisms of chronic CHIKV disease pathogenesis will inform selection and development of the most effective treatments.

\section{Acknowledgements}

The authors would like to thank MS Diamond, TS Dermody, and MT Heise for critical readings of this article.

\section{Financial \& competing interests disclosure}

Work in the Morrison laboratory is supported by NIH-NIAID grants U19 AI109680 and R01 AI108725. The authors have no other relevant affliations or financial involvement with any organization or entity with a financial interest in or financial conflict with the subject matter or materials discussed in the manuscript apart from those disclosed.

No writing assistance was utilized in the production of this manuscript.

\section{References}

1 Leo YS, Chow ALP, Tan LK, Lye DC, Lin L, Ng LC. Chikungunya outbreak, Singapore, 2008. Emerg. Infect. Dis. 15, 836-837 (2009).

2 Kaur P, Ponniah M, Murhekar MV et al. Chikungunya outbreak, South India, 2006. Emerg. Infect. Dis. 14, 1623-1625 (2008).

3 Josseran L, Paquet C, Zehgnoun A et al. Chikungunya disease outbreak, Reunion
Island. Emerg. Infect. Dis. 12, 1994-1995 (2006).

4 Depoortere E, Salmaso S, Pompa MG, Guglielmetti P, Coulombier D.

Chikungunya in Europe. Lancet 371, 723 (2008).

5 AbuBakar S, Sam I-C, Wong P-F, MatRahim N, Hooi P-S, Roslan N. Reemergence of endemic chikungunya,
Malaysia. Emerg. Infect. Dis. 13, 147-149 (2007).

6 Olowokure B, Francis L, Polson-Edwards K et al. The Caribbean response to chikungunya. Lancet Infect. Dis. 14, 1039-1040 (2014).

7 Borgherini G, Poubeau P, Jossaume A et al. Persistent arthralgia associated with chikungunya virus: a study of 88 adult 
patients on Reunion Island. Clin. Infect. Dis. 47, 469-475 (2008).

8 Gérardin P, Fianu A, Malvy D et al. Perceived morbidity and community burden after a chikungunya outbreak: the TELECHIK survey, a population-based cohort study. BMC Med. 9, 5 (2011).

9 Rodríguez-Morales AJ, Calvache-Benavides CE, Giraldo-Gómez J et al. Postchikungunya chronic arthralgia: results from a retrospective follow-up study of 131 cases in Tolima, Colombia. Travel Med. Infect. Dis. doi:10.1016/j.tmaid.2015.09.001 1-2 (2015) (Epub ahead of print).

10 Rodríguez-Morales AJ, Villamil-Gomez W, Merlano-Espinosa M, Simone-Kleber L. Post-chikungunya chronic arthralgia: a first retrospective follow-up study of 39 cases in Colombia. Clin. Rheumatol. doi:10.1007/ s10067-015-3041-8 (2015) (Epub ahead of print).

11 Rodriguez-Morales AJ, Cardona-Ospina JA, Villamil-Gómez W, Paniz-Mondolfi AE. How many patients with postchikungunya chronic inflammatory rheumatism can we expect in the new endemic areas of Latin America? Rheumatol. Int. 35(12) 2091-2094 (2015).

12 Gérardin P, Fianu A, Michault A et al. Predictors of chikungunya rheumatism: a prognostic survey ancillary to the
TELECHIK cohort study. Arthritis Res. Amp. Ther. 15, R9 (2013).

13 Javelle E, Ribera A, Degasne I, Gaüzere B-A, Marimoutou C, Simon F. Specific management of post-chikungunya rheumatic disorders: a retrospective study of 159 Cases in Reunion Island from 2006-2012. PLoS Negl. Trop. Dis. 9, e0003603 (2015).

14 Schilte C, Staikovsky F, Couderc T et al. Chikungunya virus-associated long-term arthralgia: a 36-month prospective longitudinal study. PLoS Negl. Trop. Dis. 7, e2137 (2013).

15 Chopra A, Anuradha V, Ghorpade R, Saluja $M$. Acute chikungunya and persistent musculoskeletal pain following the 2006 Indian epidemic: a 2-year prospective rural community study. Epidemiol. Infect. 140, 842-850 (2011).

16 Venugopalan A, Ghorpade RP, Chopra A. Cytokines in acute chikungunya. PLoS ONE 9, e111305 (2014).

17 Miner JJ, Aw-Yeang H-X, Fox JM et al. Chikungunya viral arthritis in the United States: a mimic of seronegative rheumatoid arthritis. Arthritis Amp Rheumatol. 67(5), 1214-1220 (2015).

18 Bouquillard É, Combe B. A report of 21 cases of rheumatoid arthritis following chikungunya fever. A mean follow-up of two years. Joint Bone Spine 76, 654-657 (2009).
19 Ganu MA, Ganu AS. Post-chikungunya chronic arthritis-our experience with DMARDs over two year follow up. J. Assoc. Physicians India 59, 83-86 (2011).

20 Poo Y-S, Rudd PA, Gardner J et al. Multiple immune factors are involved in controlling acute and chronic chikungunya virus infection. PLoS Negl. Trop. Dis. 8, e3354 (2014).

21 Labadie K, Larcher T, Joubert C et al. Chikungunya disease in nonhuman primates involves long-term viral persistence in macrophages. J. Clin. Invest. 120, 894-906 (2010).

22 Hawman DW, Stoermer KA, Montgomery SA et al. Chronic joint disease caused by persistent chikungunya virus infection is controlled by the adaptive immune response. J. Virol. 87, 13878-13888 (2013).

23 Hoarau JJ, Jaffar Bandjee MC, Krejbich Trotot $\mathrm{P}$ et al. Persistent chronic inflammation and infection by chikungunya arthritogenic alphavirus in spite of a robust host immune response. J. Immunol. 184, 5914-5927 (2010).

24 Ferris MT, Heise MT. Quantitative genetics in the study of virus-induced disease. $A d v$. Virus Res. 88, 193-225 (2014). 